\title{
Superhydrophobic graphene-decorated mesh gauze: recycling oils and organic solvents enhanced by large-diameter capillary action
}

\author{
Xue $\mathrm{Li}^{1 \dagger}$, Tingting Jiang ${ }^{1 \dagger}$, Xiaodie Wang ${ }^{1}$, Ziqiang Zhang ${ }^{1}, \mathrm{Yi} \mathrm{Li}^{1 *}$, Jianzhou Gui ${ }^{2}$, Ruiqing Liu ${ }^{1}$, \\ Zhendong Huang ${ }^{1}$, Xiaomiao Feng ${ }^{1}$, Lianhui Wang ${ }^{1}$, Wei Huang ${ }^{1,3}$ and Yanwen $\mathrm{Ma}^{1 *}$
}

\begin{abstract}
A novel recyclable, superhydrophobic and superoleophilic graphene-based textile sorbent was prepared for the removal of oil spills and organic pollutants, by functionalizing cotton mesh gauze with reduced graphene oxide (RGO) using dip-coating and hydrothermal reduction approach. The RGO-gauze composite textile showed efficient water/oil separation, and the amount of oil absorbed by the sorbent could be up to 11 times of its own weight. About 50( \pm 5$) \%$ absorbed oil could be recycled by mechanical squeezing and it was mainly extracted from the reserve of large-diameter capillary action. The RGO-gauze maintained high structural stability and outstanding recyclability after eight cycles of oil removal. This RGO-gauze consumes a small quantity of graphene $\left(0.6 \mathrm{~g} \mathrm{~m}^{-2}\right)$ and can be produced in large scale, very suitable for application in the cleanup of oil spill and organic solvent effluent.
\end{abstract}

Keywords: superhydrophobic, graphene, mesh gauze, capillary action, oil removal

\section{INTRODUCTION}

Our water resources face serious threat from the spillage of oil and its derivatives during their exploration, transportation, storage and usage [1,2]. The most urgent countermeasure to deal with the emergency spread of these pollutants is collecting them from water with the assistance of sorbents [3-7]. To achieve highly efficient oil-water separation, superhydrophobic, superoleophilic and highly porous sorbent materials are developed in recent years, which involve metal meshes [8-11], textiles
[12-17], foams [18-23], membranes [24-26], aerogels and so forth $[27,28]$. Particularly, textiles are the most attractive candidates in large-scale applications, like dozens of miles of protection belt used in marine oil spillage, due to their abundance, good mechanical durability, high flexibility and some other excellent properties. Superhydrophobic textiles were generally fabricated by roughening their skeletons/surfaces with micro/nanomaterials, such as $\mathrm{ZnO}$ and $\mathrm{SiO}_{2}$ nanoparticles [29-33], layered double hydroxide microcrystals $[34,35]$, carbon naotubes and reduced graphene oxide (RGO) as modifiers [36,37]. Among these decorations, RGO is the promising one not only because of its large specific surface area and oleophilic features, but also because its graphene oxide precursor behaves like macromolecules that can wrap each fiber in yarns by self-assembly. In addition, RGOs themselves in foam and aerogel structures have demonstrated remarkable performance in oil adsorption and recovery, although their cost, output and mechanical properties are largely behind application requirement $[38,39]$. Hence integrating textiles and RGO together to combine their advantages allows us to develop high-quality and mass production-scale sorbents.

The uptake of oil or organic solvents by porous sorbents is a complex process, probably through adsorption, absorption and capillary action mechanism [40]. In adsorption, organic molecules are in general attracted and restricted to the surface of the sorbent from the solution-adsorbent interface. Absorption is a process in which the molecules

\footnotetext{
${ }^{1}$ Key Laboratory for Organic Electronics and Information Displays \& Institute of Advanced Materials, Jiangsu Natioal Synergistic Innovation Center for Advanced Materials, Nanjing University of Posts and Telecommunications, Najing 210023, China

${ }^{2}$ School of Environmental and Chemical Engineering, Tianjin Polytechnic University, Tianjin 300387, China

${ }^{3}$ Jiangsu Singapore Joint Research Center for Organic/Bio-Electronics \& Information Displays and Institute of Advanced Materials, Nanjing Tech University, Nanjing 211816, China

$\dagger$ The first two authors contributed equally to this work.

* Corresponding authors (emails: iamyli@njupt.edu.cn (Li Y); iamywma@njupt.edu.cn (Ma Y))
} 
transport from liquid into sorbent phase and migrate beneath at least several nanometers. Capillary action occurs in pores with diameters ranging from nanometer to centimeter scale where the solute flows into the pores without the assistance of external force. The interaction force between the solute and sorbent in a capillary pore is inversely proportional to the pore diameter. Regarding oil recovery from sorbents by squeezing or twisting, small liquid-solid interaction is preferred if the sorption amount is not reduced. Hence it is of great interests to prepare oil sorbents using large pore size textiles to low liquid-solid interaction.

In this study, we selected cotton mesh gauze (warp: weft $21 \mathrm{~s} \times 32 \mathrm{~s}$ ) with average pore size of $0.6 \mathrm{~mm}$ as the substrate to prepare graphene-gauze sorbent. This gauze has downy surface because many cotton fibers protrude from yarns as illustrated in Scheme 1. The gauze was dipped into GO solution to allow GO nanosheets wrapping each fiber spontaneously. Then the dried GO-gauze was reduced in hydrothermal condition with the aid of hydrazine hydrate. The resulting $\mathrm{RGO}$-gauze replicated the template morphology well and presented superhydrophobic and superoleophilic properties as well as water-oil separation capability. The first-run sorption capacitance of RGO-gauze for soybean oil is $11 \mathrm{~g} \mathrm{~g}^{-1}$ and about $20( \pm 2) \%$ of which is ascribed to large-diameter capillary action. The capillary part that bridged by mesh could be easily recovered by squeezing. More importantly, our RGO-gauze consumes a small quantity of graphene $\left(0.6 \mathrm{~g} \mathrm{~m}^{-2}\right)$ and can be produced in large scale, very suitable for future industrial production.

\section{EXPERIMENTAL SECTION}

\section{Preparation of RGO-gauze composite}

GO aqueous dispersion was prepared as described in our previous work, whose concentration was controlled to be

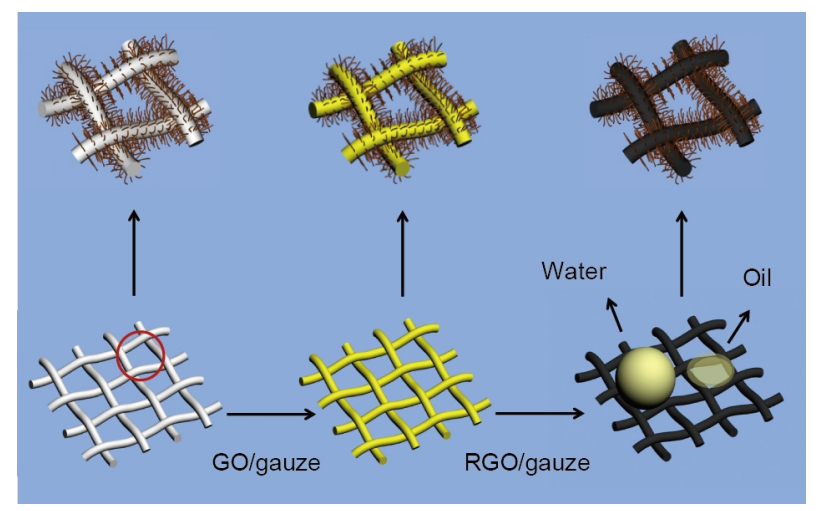

Scheme 1 The preparation method of graphene coated meshed gauze.
$1 \mathrm{mg} \mathrm{mL}^{-1}$ [41]. A piece of commercial cotton gauze (purchased from Xinxiang Hongda Textile, the yarn count was warp: weft $21 \mathrm{~s} \times 32 \mathrm{~s}$ with average pore size of $0.6 \mathrm{~mm}$ ) with area of $1.5 \mathrm{~m}^{2}$ was cleaned by sonication in deionized (DI) water before immersed into $1000 \mathrm{~mL}$ GO solution. Then the wet gauze was taken out and dried in oven at $70^{\circ} \mathrm{C}$ for $10 \mathrm{~min}$. The dip-coating process was repeated for 5 times. Then the dried GO-gauze composite was put into a 1000 $\mathrm{mL}$ Teflon-lined autoclave with $600 \mathrm{~mL}$ DI water and 10 $\mathrm{mL}$ hydrazine hydrate and then reduced at $90^{\circ} \mathrm{C}$ for $12 \mathrm{~h}$. Finally, the obtained RGO coated mesh gauze was washed with DI water for three times to remove the residuals and dried at $70^{\circ} \mathrm{C}$.

\section{Absorption capacity test}

The absorption capacity test was taken by a piece of RGOgauze $(5 \mathrm{~cm} \times 6 \mathrm{~cm})$ that was put into various organic solvents and oils until saturation. The sorption capacity $(k)$ was defined as the mass ratio of sorbed oil $\left(m_{\mathrm{ii}}\right)$ to the dry RGO-gauze $\left(m_{\mathrm{i}}\right)$.

\section{Characterization}

The products were characterized by scanning electron microscopy (SEM, Hitachi S-4800), Fourier transform infrared spectrometer (FTIR, Bruker VECTOR-22), X-ray photoelectron spectroscopy (XPS, VersaProbe PHI5000) and Raman spectroscopy (Renishaw in Via Raman Microscope with an argon-ion laser at an excitation wavelength of $523 \mathrm{~nm}$ ). Contact angle (CA) was measured with $3 \mu \mathrm{L}$ droplets of water and soybean oil using a Krüss DSA 100 (Krüss Company, Germany) apparatus at ambient temperature (The CA was measured from seven samples). The optical images were captured by a digital camera (Sony) and an optical microscope (Nikon ECLIPSE LV100N POL).

\section{RESULTS AND DISCUSSION}

Fig. 1a shows the photograph of the pristine cotton mesh gauze and RGO decorated gauze, whose sizes are $1.5 \mathrm{~m}$ $\times 1 \mathrm{~m}$. White gauze was tuned to black when RGO was coated. More interesting, the RGO-gauze could float on water surface while the raw gauze sank into water (Fig. 1b). On the RGO-gauze surface, a water droplet almost kept sphere morphology and stayed stable for a long time while an oil droplet was quickly absorbed in a few seconds (Fig. 1c). The detail wettability of the pristine and RGO-decorated gauze was analyzed by water contact angle measurement, given in Figs 1d and e. Quite different wetting processes were observed for these two gauzes. Pristine gauze is hydrophilic and can absorb water quickly. In contrast, 

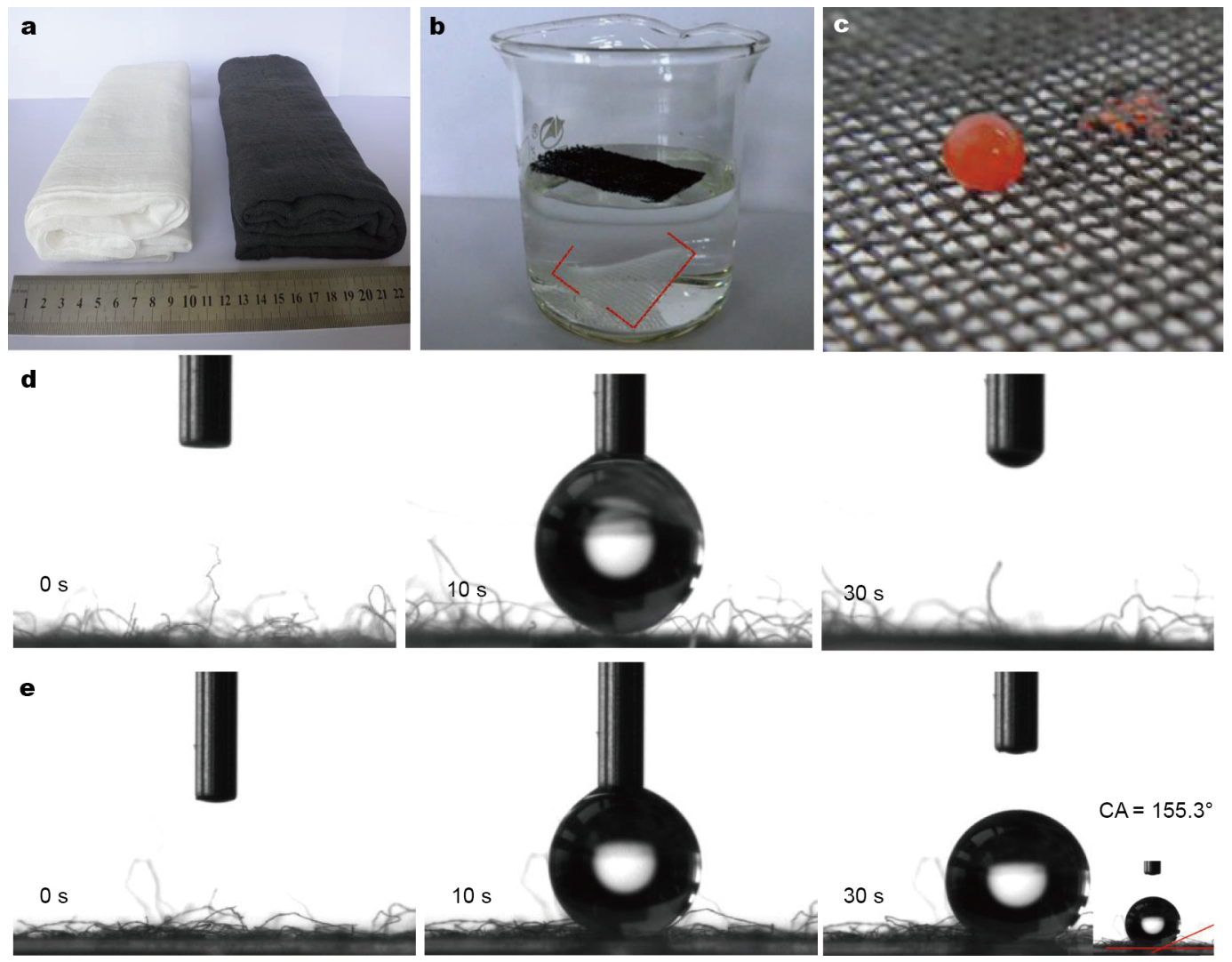

Figure 1 (a) Photograph of the gauze and RGO-gauze with size of $1.5 \mathrm{~m} \times 1 \mathrm{~m}$; (b) original gauze (down) and RGO-gauze (up) after being placed in water; (c) a water droplet (left) and an oil droplet (right) dyed with Sudan II on the RGO-gauze; (d and e) video snapshots of the contact angle of a water droplet placed onto the surface of gauze and RGO-gauze.

RGO-gauze is superhydrophobic with contact angle of $155.3( \pm 0.3)^{\circ}$. In order to understanding the role of RGO, the same treatment was also carried out for a neat cotton gauze as that for GO-gauze. The FTIR, oil and water adsorption tests clearly indicate that without RGO coating, the gauze features the unchanged surficial functional groups and wettability before and after the hydrazine treatment. All these results indicate that after RGO coating, gauze is tuned from hydrophilicity to superhydrophobicity (Fig. S1, Supplementary information). In addition, the superhydrophobic RGO-gauze also shows superoleophilic property (Fig. S2) as expected, which favors its application in oil/water separation.

The microstructure of the gauze before and after RGO coating was observed by SEM. Figs $2 a-c$ show the SEM images of the pristine gauze. It is seen that the meshes in gauze are knitted from warp and weft yarns with pore size of 0.6 $\mathrm{mm}$. Each yarn is composed of dozens of loosely twisted fibers with diameter of $10-15 \mu \mathrm{m}$ so that they produce bulk and space among the fibers. Specifically, the space in porous fiber bundles can entrap numerous air pockets, which is necessary to achieve surface superhydrophobicity. After RGO coating, the structure of gauze does not show any obvious change (Figs $2 \mathrm{~d}-\mathrm{e}$ ). But for fibers, their surfaces become rough because of the wrinkles of RGO sheets that inherits from GO (Fig. S3). The Brunauer-Emmett-Teller (BET) surface areas of the pristine gauze and RGO-gauze are 3.24 and $4.69 \mathrm{~m}^{2} \mathrm{~g}^{-1}$, indicating that the surface area is increased by RGO decorating. A significant change after RGO coating is that the hydrophilic hydroxyl groups on cellulose fibers was screened. The FTIR spectra, Raman spectra and XPS analysis (Figs S4 and S5) clearly indicate that RGO on fibers with little amount of oxygen containing groups was obtained, forming a hydrophobic surface. The water contact angle of flat RGO/glass film is $70.2( \pm 0.2)^{\circ}$ (Fig. S6). Hence the superhydrophobic features owned by RGO-gauze should result from the synergic effect of porous fiber bundles and their hydrophobic surface.

To understand the sorption capacity of RGO-gauze, several kinds of oils and organic solvents were evaluated in this study, including pump oil, soybean oil, diesel oil, glycol, 

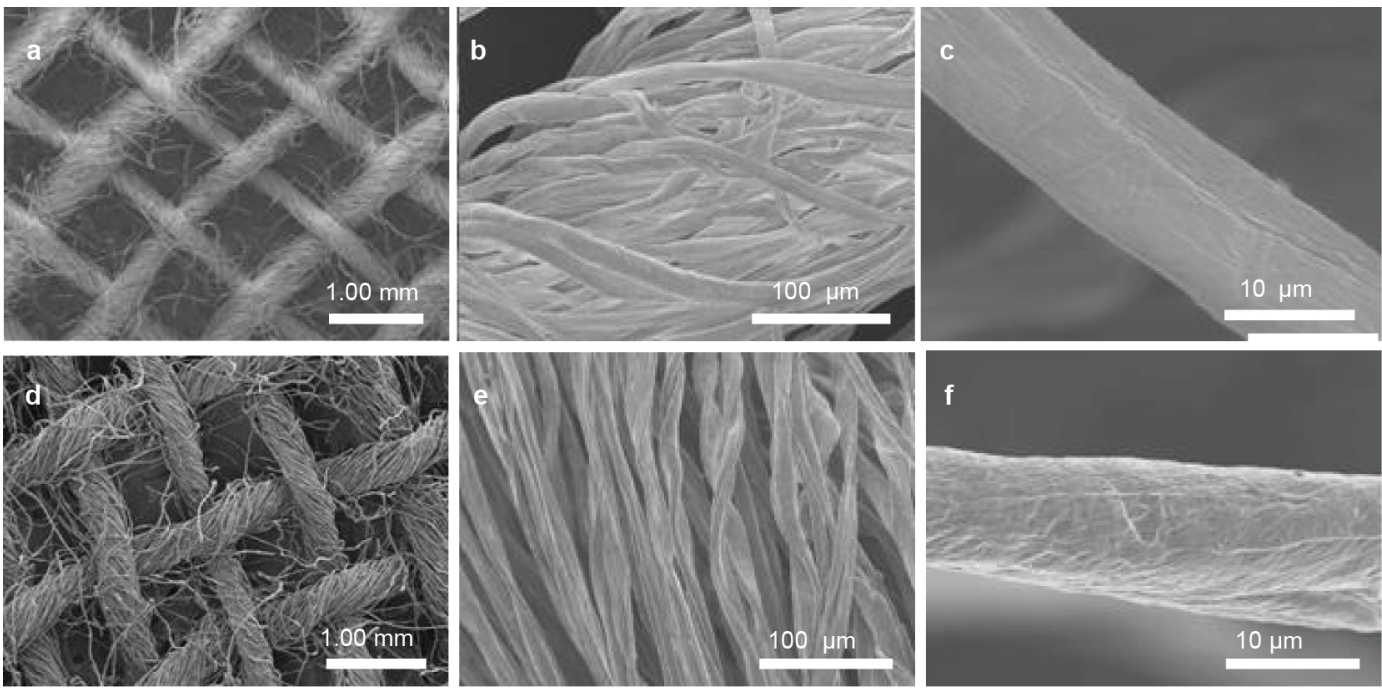

Figure 2 SEM images of the gauze (a, b and c) and RGO-gauze (d, e and f). (b) and (e) are SEM images of fibers in gauze and RGO-gauze, respectively. $(c)$ and $(f)$ are the corresponding enlarged images of $(b)$ and $(e)$.

a

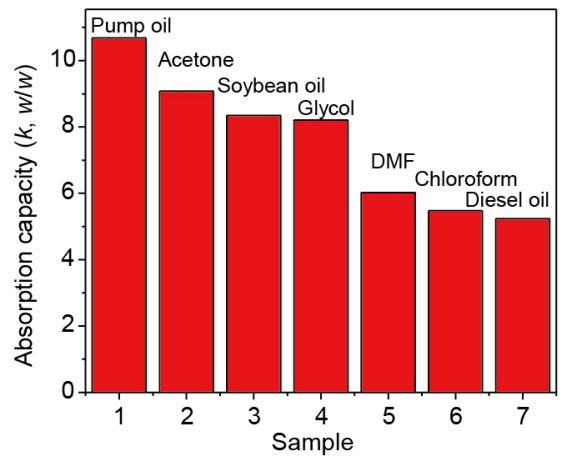

b

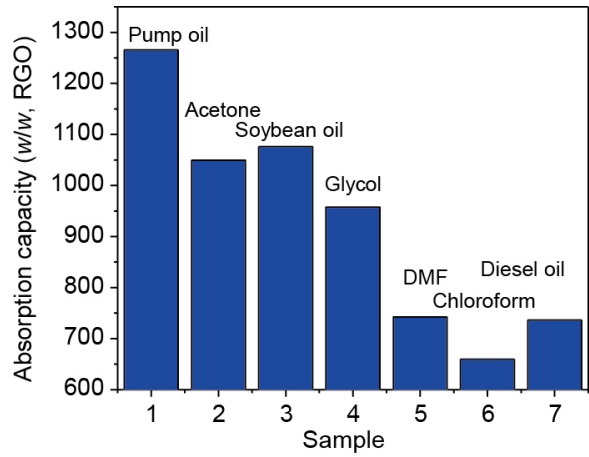

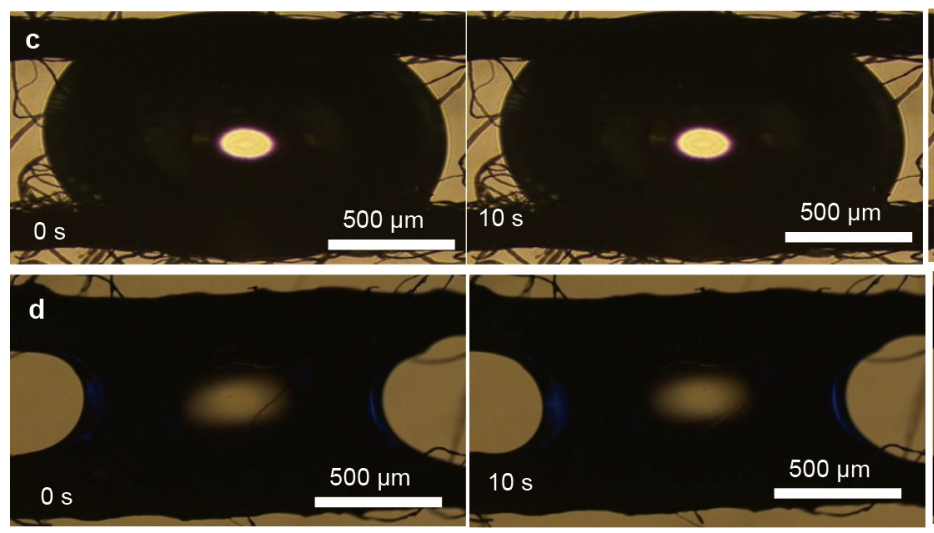
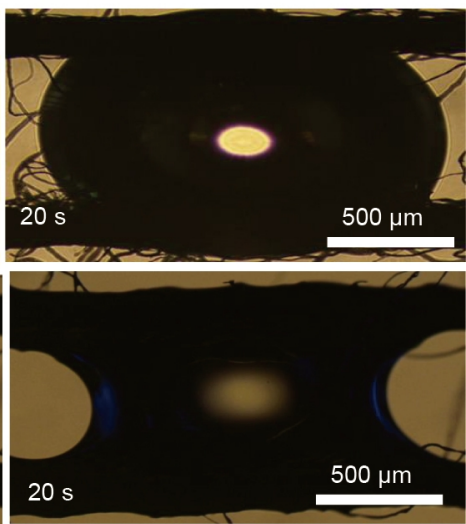

Figure 3 (a) The absorption capacity of RGO-gauze on different oils and organic solvents; (b) absorption capacity calibrated by the mass loading of RGO; (c and d) optical microscope images of capillary action of water (c) and soybean oil droplet (d) within two parallel RGO-decorated yarns.

acetone, dimethyl formamide (DMF) and chloroform. The RGO-gauze can efficiently recover oil or insoluble organic solvents from water, indicating the good oil/water absorption selectivity (Fig. S7). In general, RGO-gauze sorbs the liquids at 5 to 11 times of its own weight (Fig. 3a). Since the loading mass of RGO was as little as $0.6 \mathrm{~g} \mathrm{~m}^{-2}$, the sorption efficiency calibrated by graphene will be as high as 650 to 1300 times to the weight of the RGO (Fig. 3b), much greater than neat carbon-based materials $[26,32,33,42-46]$. As mentioned above, capillary action plays a main role in 
oil sorption. This could be well understood by the following demonstration. Figs $3 \mathrm{c}$ and d show the capillary of water and soybean oil on two parallel yarns that extracted from RGO-gauze. The space of these two parallel yarns was controlled to be $600 \mu \mathrm{m}$. The liquid droplets were bridged by the parallel yarns. The water droplet is in convex meniscus and none penetrates into the superhydrophobic fibers. In contrast, a large part of soybean oil droplet is absorbed into the fiber bundles and the remained generate concave meniscus between the yarns. To roughly evaluate the amount of oil taken by capillary from the large pores of RGO-gauze, a piece of RGO-gauze $(2 \mathrm{~cm} \times 2 \mathrm{~cm})$ was disassembled to separated yarns (Fig. S8). By calculating the difference of sorption amount between the unbroken gauze and separated yarns, the oil uptake contributed from large-diameter capillary is about $20( \pm 2) \%$.

The recyclability of oil and reusability of absorbents are important properties required for oil recovery. The recycling oils and organic solvents as well as the regeneration of RGO-gauze are illustrated in Figs $4 \mathrm{a}$ and b by squeezing and acetone washing regeneration, respectively. For these small molecule organic solvents such as acetone and DMF, the removal efficiency of RGO-gauze almost keeps the same as the fresh one due to the self-regeneration by solvent evaporation. But for those oils with large viscosity and high evaporation temperature, only about $50( \pm 5) \%$ absorbed oils
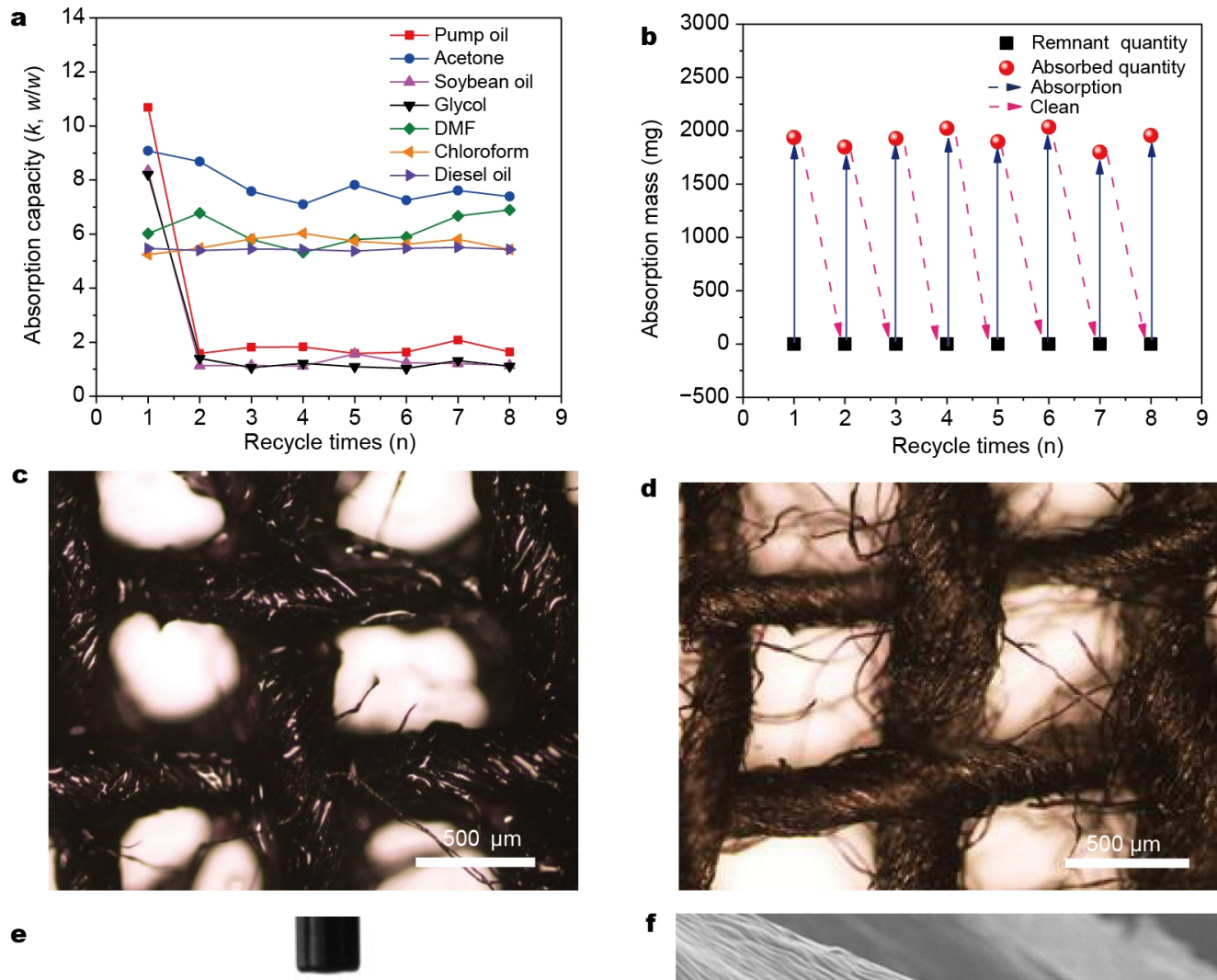

d
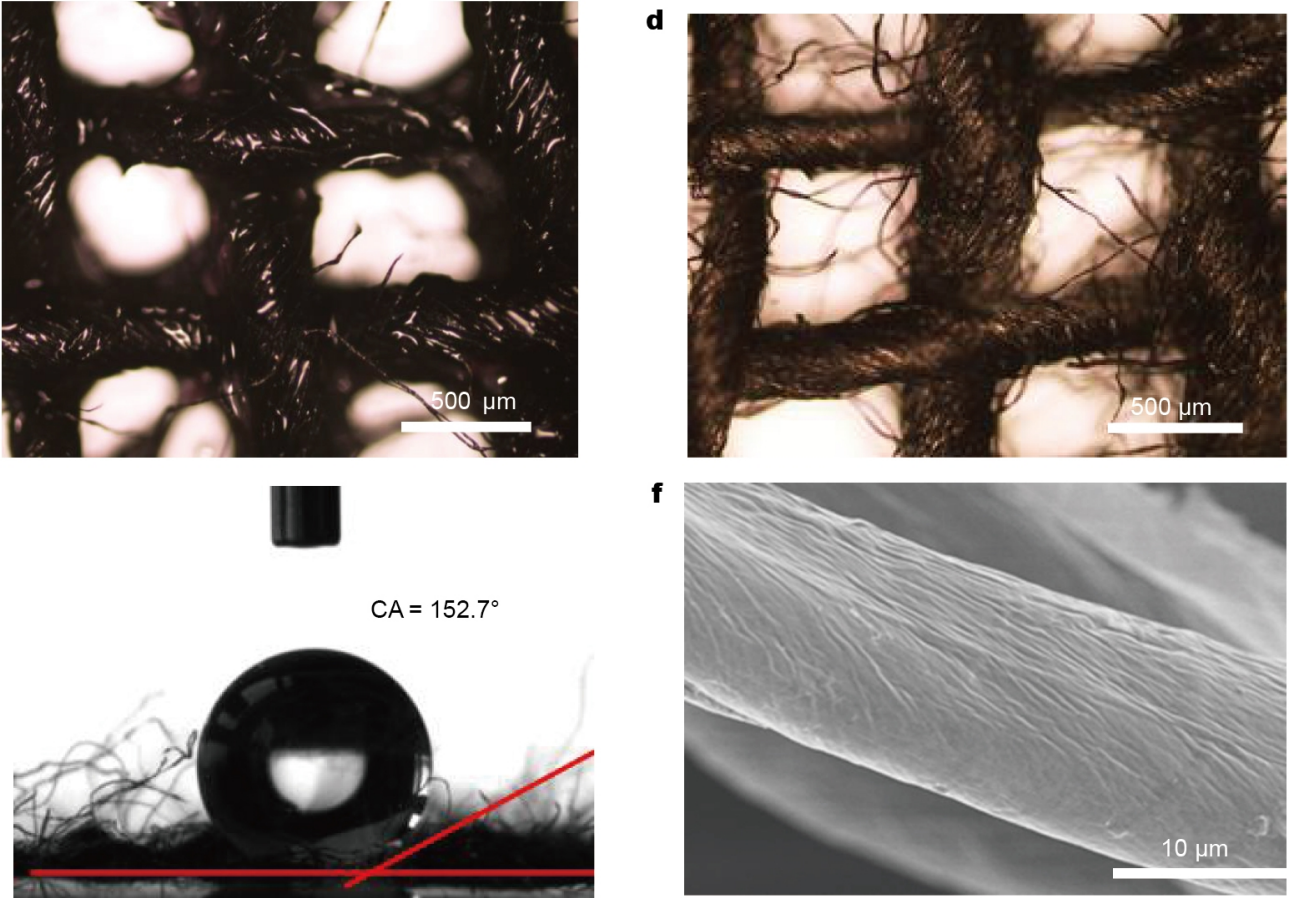

Figure 4 (a) Recyclability of RGO-gauze; (b) repeat runs of soybean oil removal by acetone washing regeneration; (c and d) optical microscope images of RGO-gauze with the sorption of soybean oil before (c) and after squeezing (d); (e) contact angle of RGO-gauze after 8 times cycling tests; (f) SEM image of the RGO coated fibers in the regenerated RGO-gauze. 
can be recovered by mechanical squeezing. The oil recovery efficiency also keeps constant of $50( \pm 5) \%$ in recycling use tests. Figs $4 \mathrm{c}$ and $\mathrm{d}$ show the photographs of soybean oil saturated RGO-gauzed before and after mechanical squeezing. It is seen that the oil in holes is removed by squeezing and the yarns could be easily distinguished, suggesting that a large amount of recycling oil should come from that kept by capillary action. Indeed, the oil uptake by large-diameter capillary makes the oil recovery easy, which allows RGO-guaze suitable for practical application. The full regeneration of the used RGO-gauze could be achieved by washing them in acetone. The absorption ability of RGO-gauze remained essentially identical after 8 cycles of testing. In addition, the wettability of the clean RGO-gauze still keeps superhdrophobicity with contact angle of $152.7( \pm 0.5)^{\circ}$ (Fig. 4e) and each fiber is well coated by RGO (Fig. 4f), indicating the outstanding mechanical stability owned by this absorbent.

\section{CONCLUSIONS}

Large scale superhydrophobic and superoleophilic RGOgauze was developed by taking advantages of the porous fiber bundles of gauze and hydrophobic property of RGO coating. The RGO-gauze delivered excellent oil absorption properties and recyclability, structural flexibility and robustness, and little graphene consumption $\left(0.6 \mathrm{~g} \mathrm{~m}^{-2}\right)$. This sorbent can selectively remove oils and insoluble organic solvents from water. About $20( \pm 2) \%$ oil uptake comes from the capillary action of the large-diameter holes in gauze mesh, which allows $50( \pm 5) \%$ absorbed oils that can be recovered by mechanical squeezing. Moreover, the efficiency was retained over several cycles of the absorption/squeezing process. Our results indicate that the advanced textile sorbent not only using the adsorption from the superhydrophobic yarns but also employing the capillary action of holes in mesh. The high-performance RGO-gauze offers great potential for treatments of oil spills and industrial effluent.

Received 27 April 2016; accepted 3 June 2016; published online 20 July 2016

1 Shannon MA, Bohn PW, Elimelech M, et al. Science and technology for water purification in the coming decades. Nature, 2008, 452: $301-310$

2 Kota AK, Kwon G, Choi W, et al. Hygro-responsive membranes for effective oil-water separation. Nat Commun, 2012, 3: 1025-1032

3 Bastani D, Safekordi AA, Alihosseini A, et al. Study of oil sorption by expanded perlite at $298.15 \mathrm{~K}$. Separ Purif Tech, 2006, 52: 295-300

4 Annunciado TR, Sydenstricker THD, Amico SC. Experimental investigation of various vegetable fibers as sorbent materials for oil spills. Mar Pollut Bull, 2005, 50: 1340-1346
5 Bayat A, Aghamiri SF, Moheb A, et al. Oil spill cleanup from sea water by sorbent materials. Chem Eng Tech, 2005, 28: 1525-1528

6 Li A, Sun HX, Tan DZ, et al. Superhydrophobic conjugated microporous polymers for separation and adsorption. Energy Environ Sci, 2011, 4: 2062-2065

7 Farag RK, El-Saeed SM. Synthesis and characterization of oil sorbers based on docosanyl acrylate and methacrylates copolymers. J Appl Polym Sci, 2008, 109: 3704-3713

8 Xue Z, Wang S, Lin L, et al. A novel superhydrophilic and underwater superoleophobic hydrogel-coated mesh for oil/water separation. Adv Mater, 2011, 23: 4270-4273

9 Song J, Huang S, Lu Y, et al. Self-driven one-step oil removal from oil spill on water via selective-wettability steel mesh. ACS Appl Mater Interfaces, 2014, 6: 19858-19865

10 Crick CR, Gibbins JA, Parkin IP. Superhydrophobic polymer-coated copper-mesh; membranes for highly efficient oil-water separation. J Mater Chem A, 2013, 1: 5943-5948

11 Liu X, Wang Y, Chen Z, et al. A self-modification approach toward transparent superhydrophobic glass for rainproofing and superhydrophobic fiberglass mesh for oil-water separation. Appl Surface Sci, 2016, 360: 789-797

12 Liu X, Ge L, Li W, et al. Layered double hydroxide functionalized textile for effective oil/water separation and selective oil adsorption. ACS Appl Mater Interfaces, 2015, 7: 791-800

13 Wu L, Zhang J, Li B, et al. Mechanical- and oil-durable superhydrophobic polyester materials for selective oil absorption and oil/water separation. J Colloid Interface Sci, 2014, 413: 112-117

14 Wang B, Li J, Wang G, et al. Methodology for robust superhydrophobic fabrics and sponges from in situ growth of transition metal/metal oxide nanocrystals with thiol modification and their applications in oil/water separation. ACS Appl Mater Interfaces, 2013, 5: 1827-1839

15 Yue G, Ma X, Zhang W, et al. A highly efficient flexible dye-sensitized solar cell based on nickel sulfide/platinum/titanium counter electrode. Nanoscale Res Lett, 2015, 10: 1-22

16 Yu M, Li W, Wang Z, et al. Covalent immobilization of metal-organic frameworks onto the surface of nylon-a new approach to the functionalization and coloration of textiles. Sci Rep, 2016, 6: 22796

17 Wang J, Geng G. Simple and eco-friendly fabrication of superhydrophobic textile for oil/water separation. Environ Tech, 2015, 37: 1591-1596

18 Zhou X, Zhang Z, Xu X, et al. Robust and durable superhydrophobic cotton fabrics for oil/water separation. ACS Appl Mater Interfaces, 2013, 5: 7208-7214

19 Dong X, Chen J, Ma Y, et al. Superhydrophobic and superoleophilic hybrid foam of graphene and carbon nanotube for selective removal of oils or organic solvents from the surface of water. Chem Commun, 2012, 48: 10660-10662

20 Huang S. Mussel-inspired one-step copolymerization to engineer hierarchically structured surface with superhydrophobic properties for removing oil from water. ACS Appl Mater Interfaces, 2014, 6: $17144-17150$

21 Pham VH, Dickerson JH. Superhydrophobic silanized melamine sponges as high efficiency oil absorbent materials. ACS Appl Mater Interfaces, 2014, 6: 14181-14188

22 Liu T, Zhao G, Zhang W, et al. The preparation of superhydrophobic graphene/melamine composite sponge applied in treatment of oil pollution. J Porous Mater, 2015, 22: 1573-1580

23 Wang $\mathrm{G}$, Zeng $\mathrm{Z}$, Wang $\mathrm{H}$, et al. Low drag porous ship with superhydrophobic and superoleophilic surface for oil spills cleanup. ACS Appl Mater Interfaces, 2015, 7: 26184-26194 
24 Duan B, Gao H, He M, et al. Hydrophobic modification on surface of chitin sponges for highly effective separation of oil. ACS Appl Mater Interfaces, 2014, 6: 19933-19942

$25 \mathrm{Xu} \mathrm{Y,} \mathrm{Bai} \mathrm{H,} \mathrm{Lu} \mathrm{G,} \mathrm{et} \mathrm{al.} \mathrm{Flexible} \mathrm{graphene} \mathrm{films} \mathrm{via} \mathrm{the} \mathrm{filtration} \mathrm{of}$ water-soluble noncovalent functionalized graphene sheets. J Am Chem Soc, 2008, 130: 5856-5857

26 Xue CH, Li YR, Hou JL, et al. Self-roughened superhydrophobic coatings for continuous oil-water separation. J Mater Chem A, 2015, 3: 10248-10253

27 Li X, Wang M, Wang C, et al. Facile immobilization of Ag nanocluster on nanofibrous membrane for oil/water separation. ACS Appl Mater Interfaces, 2014, 6: 15272-15282

28 Bi H, Yin Z, Cao X, et al. Carbon fiber aerogel made from raw cotton: a novel, efficient and recyclable sorbent for oils and organic solvents. Adv Mater, 2013, 25: 5916-5921

29 Sun H, Xu Z, Gao C. Multifunctional, ultra-flyweight, synergistically assembled carbon aerogels. Adv Mater, 2013, 25: 2554-2560

30 Wang J, Tsuzuki T, Tang B, et al. Recyclable textiles functionalized with reduced graphene oxide@ZnO for removal of oil spills and dye pollutants. Aust J Chem, 2014, 67: 71-77

31 Zhang W, Lu X, Xin Z, et al. A self-cleaning polybenzoxazine/ $/ \mathrm{TiO}_{2}$ surface with superhydrophobicity and superoleophilicity for oil/water separation. Nanoscale, 2015, 7: 19476-19483

32 Wu L, Li L, Li B, et al. Magnetic, durable, and superhydrophobic polyurethane@ $\mathrm{Fe}_{3} \mathrm{O}_{4} @ \mathrm{SiO}_{2} @$ fluoropolymer sponges for selective oil absorption and oil/water separation. ACS Appl Mater Interfaces, 2015, 7: 4936-4946

33 Duan C, Zhu T, Guo J, et al. Smart enrichment and facile separation of oil from emulsions and mixtures by superhydrophobic/superoleophilic particles. ACS Appl Mater Interfaces, 2015, 7: 10475-10481

34 Zhang L, Wu J, Wang Y, et al. Combination of bioinspiration: a general route to superhydrophobic particles. J Am Chem Soc, 2012, 134: 9879-9881

35 Yao X, Gao J, Song Y, et al. Superoleophobic surfaces with controllable oil adhesion and their application in oil transportation. Adv Funct Mater, 2011, 21: 4270-4276

36 Liu N, Cao Y, Lin X, et al. A facile solvent-manipulated mesh for reversible oil/water separation. ACS Appl Mater Interfaces, 2014, 6: $12821-12826$

37 Gui X, Wei J, Wang K, et al. Carbon nanotube sponges. Adv Mater, 2010, 22: 617-621

38 Wu ZY, Li C, Liang HW, et al. Ultralight, flexible, and fire-resistant carbon nanofiber aerogels from bacterial cellulose. Angew Chem
Int Ed, 2013, 52: 2925-2929

$39 \mathrm{Hu} \mathrm{H}$, Zhao Z, Gogotsi Y, et al. Compressible carbon nanotube-graphene hybrid aerogels with superhydrophobicity and superoleophilicity for oil sorption. Environ Sci Tech Lett, 2014, 1: 214-220

$40 \mathrm{Bi} \mathrm{H}, \mathrm{Xie} \mathrm{X}$, Yin K, et al. Spongy graphene as a highly efficient and recyclable sorbent for oils and organic solvents. Adv Funct Mater, 2012, 22: 4421-4425

41 Singh V, Jinka S, Hake K, et al. Novel natural sorbent for oil spill cleanup. Ind Eng Chem Res, 2014, 53: 11954-11961

42 Ma Y, Sun L, Huang W, et al. Three-dimensional nitrogen-doped carbon nanotubes/graphene structure used as a metal-free electrocatalyst for the oxygen reduction reaction. J Phys Chem C, 2011, 115: 24592-24597

43 Ge B, Zhang Z, Zhu X, et al. A graphene coated cotton for oil/water separation. Composites Sci Tech, 2014, 102: 100-105

44 Bi H, Huang X, Wu X, et al. Carbon microbelt aerogel prepared by waste paper: an efficient and recyclable sorbent for oils and organic solvents. Small, 2014, 10: 3544-3550

45 Zhao J, Ren W, Cheng HM. Graphene sponge for efficient and repeatable adsorption and desorption of water contaminations. J Mater Chem, 2012, 22: 20197-20202

46 Zhao Y, Hu C, Hu Y, et al. A versatile, ultralight, nitrogen-doped graphene framework. Angew Chem, 2012, 124: 11533-11537

Acknowledgments This work was jointly supported by the National Natural Science Foundation of China (61504062 and 21576211), Priority Academic Program Development of Jiangsu Higher Education Institutions (YX03001), Jiangsu National Synergetic Innovation Center for Advanced Materials (SICAM), Synergistic Innovation Center for Organic Electronics and Information Displays, Jiangsu Provincial Natural Science Foundation (BK20141424 and BK20150863), Qing Lan Project of Jiangsu Province, and the Scientific Research Foundation of Nanjing University of Posts \& Telecommunications (NY214183).

Author contributions $\mathrm{Ma}$ Y and Li Y conceived and supervised the project; Li X, Jiang T, Wang $X$ and Zhang $Z$ designed and carried out the experiments; Li X, Gui J, Liu R, Huang Z, Feng X, Li Y, Wang L, Huang W and $\mathrm{Ma} \mathrm{Y}$ analyzed the data and prepared the manuscript. All the authors participated in discussions of the research.

Conflict of interest The authors declare that they have no conflict of interest.

Supplementary information Supporting data are available in the online version of the paper. 

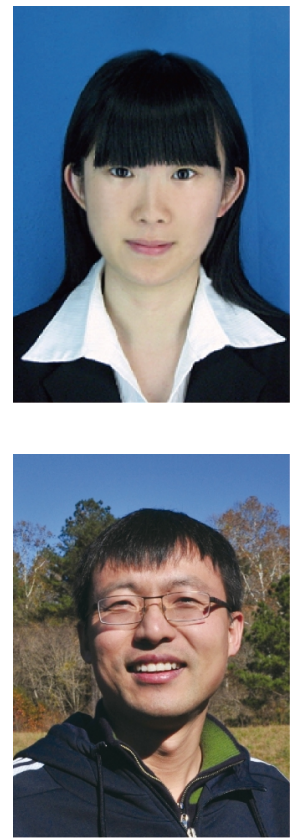

Xue Li is currently an undergraduate student majoring in material science at Nanjing University of Posts and Telecommunications (NUPT). Her research interest focuses on nanomaterials for environmental protection.
Yanwen Ma is currently a professor at the Institute of Advanced Materials (IAM) at NUPT. He received his BSc (1999) and MSc degrees (2002) in applied chemistry from Liaoning Shihua University, China, and PhD in physical chemistry (2005) from Nanjing University. After two years' (2005-2007) postdoctoral research in materials science at Nanjing University, he joined IAM in 2007. He spent one year (2013-2014) at Duke University as a visiting scholar. His current research interests include carbon nanomaterials, flexible elelctrodes and energy storages.

\section{超疏水石墨烯修饰纱布: 通过大孔径毛细作用提高油与有机溶剂的回收能力}

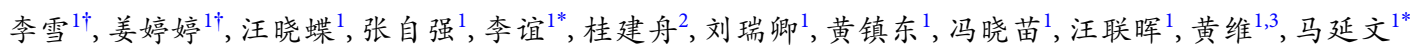

摘要 本论文采用浸渍涂覆和水热还原方法将还原氧化石墨烯沉积于棉纱布纤维表面,制备了一种新型可回收的超疏水、超亲油性石墨 烯基织物吸附材料. 该石墨烯基织物具有高效的油/水分离能力, 其吸油量可达自身质量的 11 倍以上. 约 50( \pm 5$) \%$ 的吸附油可通过机械挤压 方式回收, 其中大部分来源于石墨烯纱布网格间的大孔径毛细作用. 经过 8 次吸油/回收反复过程, 石墨烯纱布仍然具有良好的吸油能力, 表 现出高稳定性. 该方法可大规模制备石墨烯纱布, 石墨烯活性材料用量少 $\left(0.6 \mathrm{~g} \mathrm{~m}^{-2}\right)$, 特别适用于油污和有机污水的回收应用. 